\title{
NOTE
}

\section{Intrahypothalamic Implants of Testosterone or Dihydrotestosterone in Neonatal Female Rats with Reference to Induction of Sterility}

\author{
SHINJI HAYASHI \\ Endocrine Division, National Cancer Center Research Institute, \\ Tsukiji 5-chome, Chuo-ku, Tokyo 104, Japan
}

\begin{abstract}
Synopsis
Implants of paraffin micropellets containing about $5 \mu \mathrm{g} 5 \alpha$-dihydrotestosterone (DHT) into the hypothalamus of 5-day-old female rats were without effect on the sexual differentiation of the brain. By contrast, approximately the same amount of testosterone propionate (TP) given as subcutaneous or intrahypothalamic micropellets masculinized the female brain. In the light of these results as well as the author's previous findings that an antiestrogen implanted into the hypothalamus of neonatal female rats failed to block masculinization by subcutaneous injection of $T P$, the possibility cannot be excluded that testosterone is capable of masculinizing the brain of neonatal females without being converted into estrogens.
\end{abstract}

Masculinization of the brain in female rats treated neonatally with androgens or estrogens as evidenced by sterility syndrome characterized by anovulation and persistent vaginal estrus is well established (Takewaki, 1962; Gorski, 1971; Arai, 1973). Micropellets of testosterone propionate (Wagner et al., 1966; Nadler, 1968 and 1972; Hayashi and Gorski, 1974), estradiol (Hayashi, 1976a) or estradiol benzoate (Döcke and Dörner, 1975) implanted into the hypothalamus of neonatal female rats also masculinize the brain while similar pellets placed subcutaneously were without effect. This effect of testosterone has been postulated to depend upon its conversion into estrogen in the brain of female rats, since aromatizing enzyme involved in the conversion is present in the neonatal hypothalamus (Naftolin et al., 1971 and 1975; Weisz and Gibbs, 1974) and the neonatal administration of non-aromatizable androgen, e.g. 5 $\alpha$-dihydrotestosterone (DHT), fails to exert the masculinizing effect on the brain of female rats (Arai, 1972; McDonald and Doughty, 1972a and 1974; Ulrich et al., 1972; van der Schoot et al., 1976). However, in view of the finding of the present author that neonatal intrahypothalamic implants of an antiestrogen, MER-25, elicited no significant block of masculinization of the brain by the neonatal injection of TP in female rats, the concept attributing androgen-sterilization to an exclusively estrogen function must be accepted with some reservation. In this connection, it was thought of some interest to study whether intrahypothalamic implants of non-aromatizable androgen in neonatal female rats could cause masculinization of the brain. The results of the experiments are dealt with in this paper. 


\section{Materials and Methods}

Seventy-three female rats of the Sprague-Dawley strain received either intrahypothalamic or subcutaneous implantation of two paraffin pellets each containing $1 \%$ or $10 \%$ testosterone propionate (TP), or $10 \%$ DHT at 5 days of age (day of birth=day 1$)$. The method of intracranial implantation of micropellets was the same as that described previously (Hayashi, 1974, $1976 \mathrm{a}$ and b). Five intact animals served as controls. The micropellets containing either $\mathrm{TP}+$ carbon marker or $\mathrm{DHT}+$ carbon marker were weighed by an ultramicrobalance (Mettler, ME22) to the nearest $0.1 \mu \mathrm{g}$. Six control micropellets containing $1 \%$ TP, $10 \%$ TP and $10 \%$ DHT weighed $17.7 \pm 0.5,22.8 \pm 1.1$ and $26.6 \pm 0.6 \mu \mathrm{g}$ (mean \pm S.E.), respectively. Since each animal received two pellets, the amount of steroids given to each pup averaged 0.35 or $4.5 \mu \mathrm{g} \mathrm{TP}$, or $5.3 \mu \mathrm{g} \mathrm{DHT}$.

The operated pups were returned to their nursing mother immediately after recovery from anesthesia and weaned at 22 days of age. Daily vaginal smears were taken after vaginal opening until the day of sacrifice. The right ovary was removed and weighed at 90 days of age in rats which showed persistent estrus continuing more than 20 consecutive days and at 120 days of age in the rest of the animals. All animals were killed 3 weeks later. Brains were perfused with $10 \%$ buffered formalin, fixed in the same solution, cut frozen in gelatin at $60 \mu \mathrm{m}$ and stained with carbol fuchsin. The localization of the intrahypothalamic micropellets was determined histologically. Ovaries, adrenals, thyroids, pituitary and uterus were weighed, fixed in Bouin's solution, cut in paraffin at $10 \mu \mathrm{m}$ and stained with Delafield's hematoxylin and eosin. The presence or absence of corpora lutea (CL) was checked in each animal. Those rats which showed persistent vaginal cornification or had no $\mathrm{CL}$ at sacrifice were determined sterile.

\section{Results}

The micropellets were found in medial preoptic area (pom), anterior hypothalamic area (ha), paraventricular nucleus (fm), dorsomedial and ventromedial hypothalamic nucleus (hdm and hvm) or arcuate nucleus (ar) (Fig. 1). All rats bearing bilateral $10 \%$ TP-paraffin pellets in the hypothalamus (Fig. 1B) had no CL in their ovaries at 90 days of age, while 3 of 8 rats given two micropellets of $10 \%$ TP-paraffin mixture

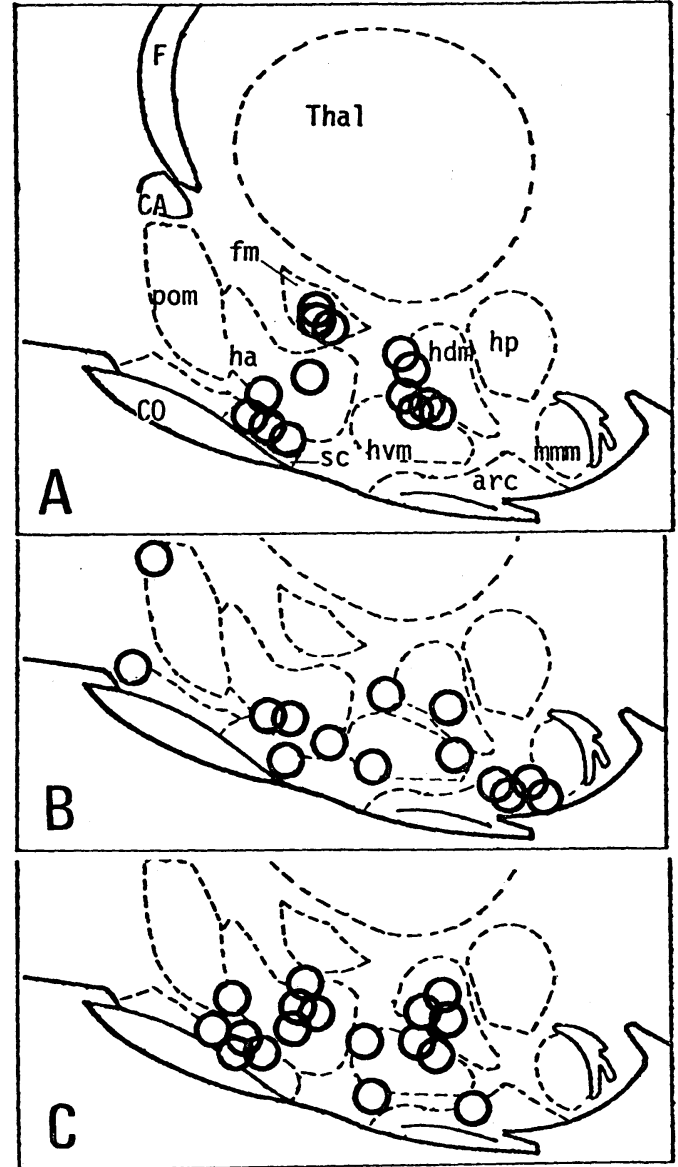

Fig. 1. Parasagital diagrams dipicting loci of paraffin micropellets containing 1\% TP (A), 10\% TP (B) and $10 \%$ DHT $(\mathrm{C})$, respectively. Abbreviations: arc, arcuate nucleus; $\mathrm{CA}$, anterior commissure; $\mathrm{CO}$, optic chiasma ; F, fornix ; fm, paraventricular nucleus; ha, anterior hypothalamic area; hdm, dorsomedial hypothalamic nucleus; hp, posterior hypothalamic nucleus; hvm, ventromedial hypothalamic nucleus; mmm, medial mamillary nucleus; pom, medial preoptic area; sc, suprachiasmatic nucleus; Thal, thalamus.

subcutaneously had CL at 120 days of age and 2 of them still at sacrifice performed at 141 days (Table 1). On the contrary, animals given $1 \% \mathrm{TP}$ - or $10 \%$ DHT-paraffin micropellets invariably had $\mathrm{CL}$ at 120 days of age without reference to locus of the pellets (Table 1, Fig. 1A, C). 
Table 1. 1ncidence of sterility in rats given micropellets of $1 \%$ TP-, $10 \%$ TP- or $10 \%$ DHTparaffin mixture intracranially (IC) or subcuaneously (SC) at 5 days of age.

\begin{tabular}{|c|c|c|c|c|c|c|c|c|c|c|}
\hline \multirow{3}{*}{ Group } & \multirow{3}{*}{$\begin{array}{l}\text { Total } \\
\text { no. of } \\
\text { rats }\end{array}$} & \multicolumn{9}{|c|}{ Incidence of sterility } \\
\hline & & \multicolumn{3}{|c|}{$\begin{array}{l}\text { At } 45 \text { days } \\
\text { of age }\end{array}$} & \multicolumn{3}{|c|}{$\begin{array}{l}\text { At } 90 \text { or } 120 \\
\text { days of age }\end{array}$} & \multicolumn{3}{|c|}{ At sacrifice } \\
\hline & & $\mathrm{CL}+$ & $\mathrm{CL}-$ & $\%$ & $\mathrm{CL}+$ & $\mathrm{CL}-$ & $\%$ & $\mathrm{CL}+$ & $\mathrm{CL}-$ & $\%$ \\
\hline Intact & 5 & 5 & 0 & 0 & 5 & 0 & 0 & 5 & 0 & 0 \\
\hline $1 \%$ TP SC & 6 & 3 & 3 & 50 & 6 & 0 & 0 & 6 & 0 & 0 \\
\hline IC & 14 & 13 & 1 & 7 & 14 & 0 & 0 & 14 & 0 & 0 \\
\hline $10 \%$ TP SC & 8 & 6 & 2 & 25 & 3 & 5 & $63 a$ & 2 & 6 & 75 \\
\hline IC & 14 & 12 & 2 & 14 & 0 & 14 & $100 \mathrm{~b}$ & 0 & 14 & 100 \\
\hline $10 \%$ DHT SC & 6 & 5 & 1 & 17 & 6 & 0 & 0 & 6 & 0 & 0 \\
\hline IC & 17 & 17 & 0 & 0 & 17 & 0 & 0 & 17 & 0 & 0 \\
\hline
\end{tabular}

$\mathrm{CL}+$ and $\mathrm{CL}-$ : No. of rats with and without corpora lutea, respectively.

a $v s$ b: Significantly different by Fisher's exact probability test $(\mathrm{p}<0.05)$.

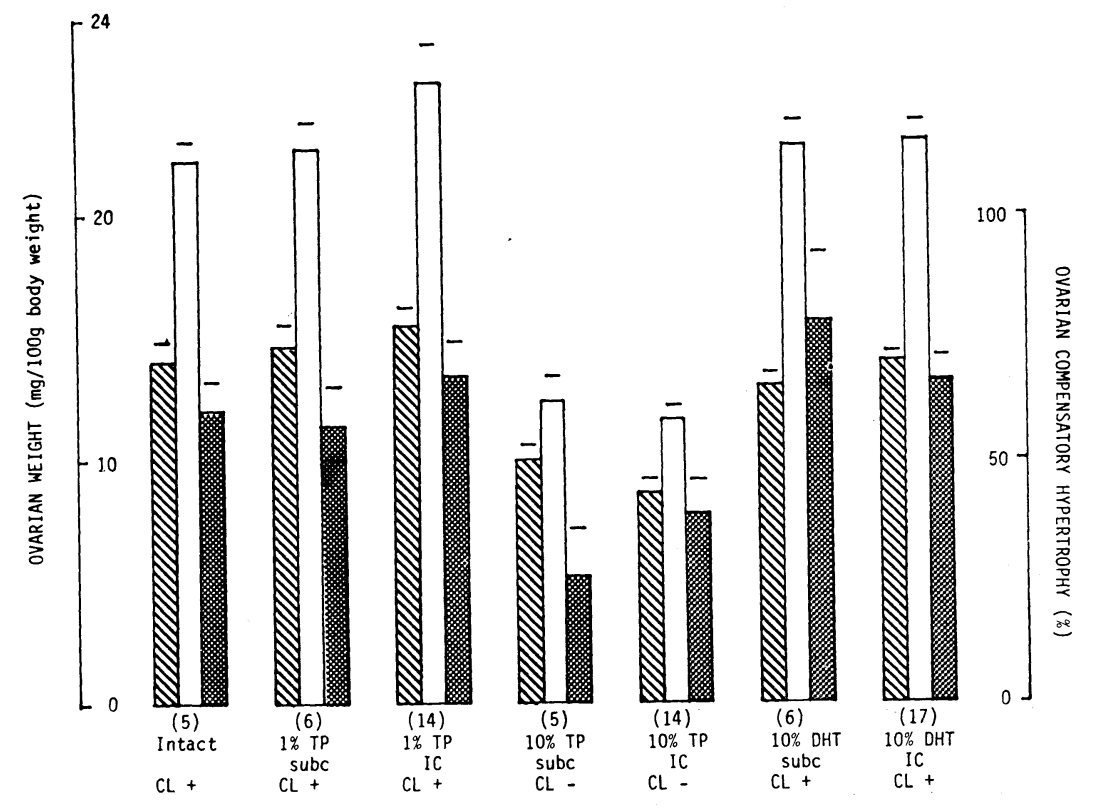

Fig. 2. Ovarian weight (mg/100 g body weight) and ovarian compensatory hypertrophy (\%). Hatched, blank and dotted bars indicate weights of right and left ovaries and ovarian compensatory hypertrophy, respectively. Lines above the bars indicate standard errors of means. The number of rats is given in parentheses. Ovarian compensatory hypertrophy was calculated by the following formula: $\frac{\text { Left ovarian weight }- \text { right ovarian weight }}{\text { right ovarian weight }} \times 100 \%$

The mean weights of the right and left ovaries of the rats with CL were 13-16 and $22-26 \mathrm{mg} / 100 \mathrm{~g}$ body weight, respectively, while those of the rats without $\mathrm{CL}$ were $8-10$ and $11-14 \mathrm{mg} / 100 \mathrm{~g}$ body weight, respectively, regardless of the treatment the animals had received (Fig. 2). The mean ovarian compensatory hypertrophy in rats sterilized by subcutaneous implantation of $10 \%$ TP micropellets was $26 \%$, being significantly smaller in magnitude than in intact controls (60\%) (Fig. 2). Adrenals, 
thyroids, pituitary and uterus were not significantly different in weight in the experimental groups from in the intact controls (data not shown).

\section{Discussion}

Subcutaneous injections of DHT or its propionate (DHTP) in neonatal female rats were reported to be without effect (McDonald and Doughty, 1972a) or only weakly effective in masculinizing the brain (Gerall et al., 1975; van der Schoot et al., 1976). The present experiments revealed that about $5 \mu \mathrm{g}$ of DHT placed in the hypothalamus of neonatal female rats exerted no masculinizing effect on the brain, while hypothalamic implants of approximately the same amount of TP invariably resulted in sterility. Thus, it was ascertained that DHT is incapable of masculinizing the female brain at least with respect to the mode of gonadotropin secretion. Five of 8 rats $(63 \%)$ given 10\% TP subcutaneously were found sterile at 90 or 120 days of age, while all of 14 rats given the same amount of TP intracranially became sterilized (Table 1). Since the difference in the values between these two groups was significant $(p<0.05)$, it is evident that TP applied intracranially was highly effective in masculinizing the brain. Moreover, it is worthy of mention that subcutaneous micropellets containing about $5 \mu \mathrm{g}$ TP (i.e. 10\% TP) conduced to sterility, whereas the same amount of DHT (i.e. $10 \%$ DHT) placed in the hypothalamus failed to evoke the brain masculinization. Therefore, the masculinizing capacity of TP pellets was at least 10 times greater than that of DHT pellets.

The effects of DHT (or DHTP) suppressing gonadotropin secretion have been demonstrated by several authors in neonatal and weaned rats. Van der Schoot et al. (1976) reported that serum levels of luteinizing hormone and follicle stimulating hormone were lowered in neonatal males castrated on day 1 and killed $48 \mathrm{hr}$ after DHTP injection. Decrease in serum gonadotropins was also reported by Korenbrot et al. (1975) in neonatal, castrated males and intact females $48 \mathrm{hr}$ after DHTP injection and by Swerdloff et al. (1972) and Naftolin and Feder (1973) in immature and mature rats. However, the suppressive effects of DHT on the secretion of gonadotropins during the neonatal period may be independent of the mechanism involved in the masculinization of the brain.

The finding of McDonald and Doughty (1972b and 1973/74) that the antiestrogen, MER-25, given by subcutaneous injection blocked the masculinizing effect of testosterone strongly supports the concept that the aromatization of androgen is required for its exertion of the masculinizing effect on the female brain. However, the experiments carried out by the present author (Hayashi, 1974 and 1976b) with intrahypothalamic implants of MER-25 in neonatal female rats given TP subcutaneously yielded results at variance with those of McDonald and Doughty. On the other hand, Kato (1976) recently found receptors for both DHT and testosterone in the cytosol fraction from the neonatal female rat brain. Taking. all those data into account, it might be safe to conclude that 1) DHT is hardly capable of masculinizing the brain in neonatal female rats; 2) estrogens produced from testosterone by aromatization in the hypothalamus can masculinize the brain if the sufficient level is reached; and 3) since the conversion rate of testosterone into estrogens in the hypothalamus and limbic system of neonatal female rats is known to be less than 1\% (Reddy et al., 1974; Naess et al., 1975; Naftolin et al., 1975), the possibility cannot be excluded that remaining testosterone plays a role in masculinizing the female brain. 


\section{Acknowledgements}

The author expresses his thanks to Miss H. Nagashima for her technical assistance and Dr. K. Takewaki, Prof. Emeritus at the University of Tokyo, for his valuable criticism and help in preparing the manuscript.

\section{References}

Arai, Y. (1972). Endocrinol. Japon. 19, 389.

Arai, Y. Neuroendocrine Control (edited by K. Yagi and S. Yoshida). Univ. Tokyo Press, Tokyo. p. 27 (1973).

Döcke, F. and G. Dörner (1975). Endokrinologie $65,375$.

Gorski, R. A. Frontiers in Neuroendocrinology (edited by L. Martini and W. F. Ganong). Oxford Univ. Press, N. Y., p. 237 (1971).

Gerall, A. A., M. M. McMurray and A. Farrell (1975). J. Endocr. 67, 439.

Hayashi, S. (1974). Endocrinol. Japon. 21, 453.

Hayashi, S. (1976a). ibid. 23, 55.

Hayashi, S. (1976b). Proc. Soc. Exp. Biol. Med. 152, 389.

Hayashi, S. and R. A. Gorski (1974). Endocrinology 94, 1161.

Kato, J. (1976). J. Steroid Biochem. 7, 1179.
Korenbrot, C. C., D. C. Paup and R. A. Gorski (1975). Endocrinology 97, 709.

McDonald, P. G. and C. Doughty (1972a). J. Reprod. Fert. 30, 55.

McDonald, P. G. and C. Doughty (1972b). J. Endocr. 55, 455.

McDonald, P. G. and C. Doughty (1973/74). Neuroendocrinology 13, 182.

McDonald, P. G. and C. Doughty (1974). J. Endocr. 61, 95.

Nadler, R. D. (1968). J. Comp. Physiol. Psychol. 66, 157.

Nadler, R. D. (1972). Neuroendocrinology 9, 349.

Naess, O., A. Attramadal and A. Aakvaag (1975). Endocrinology 96, 1.

Naftolin, F., K. J. Ryan and Z. Petro (1971). J. Clin. Endocr. Metab. 33, 368.

Naftolin, F., K. J. Ryan, I. J. Davis, V. V. Reddy, F. Flores, Z. Petro, M. Kuhn, R. J. White, Y. Takaoka and L. Wolin (1975). Rec. Prog. Horm. Res. 31, 295.

Reddy, V.V.R., F. Naftolin and K. J. Ryan (1974). Endocrinology 94, 117.

Takewaki, K. (1962). Experientia 18, 1.

Ulrich, R., A. Yuwiler and E. Geller (1972). Proc. Soc. Exp. Biol. Med. 139, 411.

Van der Schoot, P., P. D. M. van der Vaart and J. T. M. Vreeburg (1976). J.Reprod.Fert. 48, 385.

Wagner, J. W., W. Erwin and V. Critchlow (1966). Endocrinology 79, 1135.

Weisz, J. and C. Gibbs (1974). ibid. 94, 616. 